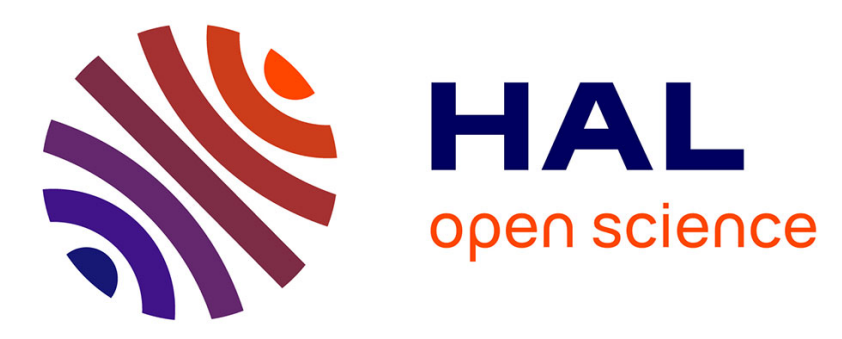

\title{
Combining a Helmholtz solver with the flame describing function to assess combustion instability in a premixed swirled combustor
}

Camilo Silva, Franck Nicoud, Thierry Schuller, Daniel Durox, Sébastien

Candel

\section{To cite this version:}

Camilo Silva, Franck Nicoud, Thierry Schuller, Daniel Durox, Sébastien Candel. Combining a Helmholtz solver with the flame describing function to assess combustion instability in a premixed swirled combustor. Combustion and Flame, 2013, 160 (9), pp.1743-1754. 10.1016/j.combustflame.2013.03.020 . hal-00854137

\section{HAL Id: hal-00854137 https://hal.science/hal-00854137}

Submitted on 26 Aug 2013

HAL is a multi-disciplinary open access archive for the deposit and dissemination of scientific research documents, whether they are published or not. The documents may come from teaching and research institutions in France or abroad, or from public or private research centers.
L'archive ouverte pluridisciplinaire HAL, est destinée au dépôt et à la diffusion de documents scientifiques de niveau recherche, publiés ou non, émanant des établissements d'enseignement et de recherche français ou étrangers, des laboratoires publics ou privés. 


\title{
Combining a Helmholtz solver with the Flame Describing Function to assess combustion instability in a premixed swirled combustor
}

\author{
C. F. Silva ${ }^{1, a}$, F. Nicoud ${ }^{2, b}$, T. Schuller ${ }^{3, c, d}$, D. Durox ${ }^{4, c, d}$, S. Candel ${ }^{5, c, d}$ \\ ${ }^{a}$ Technische Universität München D-85747 Garching, Germany \\ ${ }^{b}$ Université Montpellier II, 34095 Montpellier, France \\ ${ }^{c}$ CNRS, UPR 288 Laboratoire d'Energétique Moléculaire et Macroscopique, Combustion \\ (EM2C) Grande Voie des Vignes, 92295 Châtenay-Malabry, France \\ ${ }^{d}$ Ecole Centrale Paris, 92295 Châtenay-Malabry, France
}

\begin{abstract}
Limit cycles of combustion instabilities can be estimated by studying the nonlinear behavior of flame dynamics. In the present study the flame describing function (FDF) framework is combined with a linear acoustic Helmholtz solver in order to estimate the growth rate of the acoustic perturbations in a swirled combustor. It is assumed that when this growth rate equals the inherent dissipation of the system, acoustic oscillation amplitudes cease to grow and a stationary state, i.e., a limit cycle, is reached. In the same way, the FDF is combined with an analytical acoustic model for a quasi-1D version of the combustor. Numerical and analytical results are compared to experimental data and a reasonable agreement is obtained in terms of frequency, growth rate and amplitude of oscillations at the limit cycle.
\end{abstract}

Keywords: Combustion instabilities, Flame Describing Function (FDF), Helmholtz solver, limit cycle.

Email address: kamilosilva@gmail.com (C. F. Silva )

${ }^{1}$ Corresponding author. Post-doctoral fellow, Technische Universität München

${ }^{2}$ Professor, Université Montpellier II, I3M - CNRS UMR 5149 - CC51

${ }^{3}$ Professor, Ecole Centrale Paris and CNRS

${ }^{4}$ Professor, Ecole Centrale Paris and CNRS

${ }^{5}$ Professor, Ecole Centrale Paris and CNRS 


\section{Introduction}

Modern aeroengines and gas turbines are required to have high performances with low fuel consumption and reduced emissions. To achieve these requirements, these systems are designed to operate in a premixed mode at low equivalence ratios with the drawback to enhance the flame sensitivity to flow perturbations. Under certain conditions, the unsteady heat release produced by the turbulent flame and the acoustic fluctuations generated in the chamber may couple, giving rise to what is known as combustion instabilities. An earlier criterion for such instabilities was proposed by Rayleigh (1878), much research on these issues was carried out in the field of liquid rockets (see for example Crocco (1969)). More recent investigations are reviewed by Candel (2002). Combustion dynamics in gas turbines are the subject of a book edited by Lieuwen and Yang V., (Eds.) (2005). This phenomenon may have serious consequences, causing structural vibrations, increasing heat fluxes to the chamber walls and reducing the system life duration.

Combustion instabilities constitute an active domain of research where much attention has been focused on the physical modeling of the processes leading to oscillations. Current analytical models have been designed in order to estimate the response of flames submitted to velocity fluctuations. In many of these models it is assumed that acoustic fluctuations remain small in comparison to the mean values of the flow: acoustics is then considered linear and second order terms in the dynamical equations can be neglected. A Flame Transfer Function (FTF) is used in this framework. In its simplest definition, the FTF only depends on frequency and links the ratio between the Fourier transforms of the unsteady heat release rate fluctuations $\hat{\dot{Q}}$ and acoustic velocity fluctuations $\hat{u}$ as follows:

$$
\mathcal{F}(\omega)=G(\omega) e^{i \omega \tau}=\frac{\hat{\dot{Q}}(\omega) / \overline{\dot{Q}}}{\hat{u} / \bar{u}}
$$

where $\omega=2 \pi f$ stands for the angular frequency of the perturbation, $G$

is the gain and $\tau$ a time delay (Crocco, 1951, 1952). Here $\bar{Q}$ and $\bar{u}$ denote a mean flame power and velocity introduced for scaling purposes. The FTF can be either measured or modeled theoretically or numerically (Ducruix et al., 2000; Külsheimer and Büchner, 2002; Truffin and Poinsot, 2005; Durox et al., 2009; Huber and Polifke, 2009; Kim et al., 2010; Schuermans et al., 2011; Duchaine et al., 2011; Tay-Wo-Chong et al., 2012; Palies et al., 2011b). In a 
second step, this function can be combined to a model of the system acoustics which is often obtained analytically (Dowling and Stow, 2003; Sattelmayer and Polifke, 2003; Poinsot and Veynante, 2005). Also, the FTF can be combined to numerical tools which are used to solve the wave equation, usually expressed in the frequency domain (Roux et al., 2005; Nicoud et al., 2007; Camporeale et al., 2011). These numerical tools allow to account for the geometrical complexity of real systems and rely on Helmholtz solvers to model linear acoustics in low Mach number flows. By means of these methodologies, it is possible to estimate the frequency, structure and growth rate of the unstable acoustic modes of the system. The growth rate is exponential in linear acoustics, and since non-dissipative and only linear terms are accounted for, the amplitude of oscillations increases infinitely (Bloxsidge et al., 1988). In order to estimate acoustic amplitudes of limit cycles, nonlinear terms must be included into the modeling (Culick, 1994; Dowling, 1997; Noiray et al., 2008; Boudy et al., 2011; Palies et al., 2011a).

Modeling of nonlinearities is needed to retrieve the evolution of acoustic oscillations when the system becomes unstable. In a complete nonlinear analysis, all second (or higher) order terms should be kept. Nonlinearities associated to gas dynamics are responsible for acoustic damping, mode to mode transfer of energy and limit cycles. In this context, Culick (1994) proposed a methodology in which a Galerkin method based on an expansion of flow variables on the acoustic eigenmodes is used to convert partial differential equations into ordinary differential equations. This provides a set of second order equations of modal amplitudes. All nonlinearities are contained in the source terms. When the time derivative of the amplitude is equal to zero, a limit cycle is reached. Another approach has been proposed (Dowling, 1997) in which it is supposed that the main nonlinear terms are those representing the unsteady heat release rate associated to velocity perturbations. In many systems, it is shown that acoustics remains linear and that limit cycles are reached due to a saturation of the heat release rate or change in the phase lag between flow and heat release rate perturbations (Noiray et al., 2008).

In this framework it is possible to introduce the Flame Describing Function (FDF) concept. The FDF model accounts for the flame nonlinearity but does not represent the possible nonlinear interactions with turbulence or other flow features like the precessing vortex core (PVC) existing in swirling flows. It has been shown however that such interactions are of lesser importance to the combustion instability process (see for example Moeck et al. (2012) where such interactions are investigated). When considering the FDF 
approach, the flame response depends not only on the frequency of the acoustic velocity perturbation, but also on its amplitude (Dowling, 1997, 1999; Noiray et al., 2008; Durox et al., 2009). The FDF is then defined as

$$
\mathcal{F}(|\hat{u}|, \omega)=G(|\hat{u}|, \omega) e^{i \omega \tau}=\frac{\hat{\dot{Q}}(|\hat{u}|, \omega) / \overline{\dot{Q}}}{\hat{u} / \bar{u}}
$$

where $|\hat{u}|$ denotes the amplitude of the acoustic perturbation. Studies carried out on this basis lead to some reasonably close representations of experimental observations like mode switching, triggering, frequency shifting during transients (Noiray et al., 2008; Boudy et al., 2011). The FDF is measured experimentally and is used as an input of analytical representations of the system acoustics. The output of this quasi-analytical approach are the growth rate of the acoustic modes, their frequency of oscillation and spatial structure as a function of the perturbation amplitude level. For a given frequency and for increasing values of the acoustic velocity amplitude, the gain and phase of the FDF evolve (Balachandran et al., 2005; Bellows et al., 2007; Durox et al., 2009; Schimek et al., 2011; Kim and Hochgreb, 2011). Depending on $|\hat{u}|$, a mode may be found stable or unstable; when the growth rate $\omega_{i}$ exceeds damping at small amplitude the mode is unstable and the limit cycle corresponds to the particular value of $|\hat{u}|$ for which the growth rate tends to the damping rate. Limit cycles reached in a swirled turbulent combustor were recently estimated for different operating conditions and burner geometries by following this general framework (Palies et al., 2011a). Despite important assumptions regarding the geometry (the combustor was represented as an acoustic network of three coupled cavities), good estimation of the resonant frequencies and limit cycles were obtained, especially for configurations in which strong combustion oscillations take place.

Analytical approaches require that the geometry is simple enough to be represented by an arrangement of 1D homogeneous elements and usually rely on the compact approximation in which combustion and geometrical changes take place over regions of small dimension compared to the acoustic wavelength. This approximation is not always justified for practical industrial combustion systems such as (modern) aero-gas turbines. The purpose of the present work is to investigate how the FDF formalism can be used in a more general framework. The stability behavior is here analyzed by means of a Helmholtz solver combined with FDF methodology to represent the nonlinear response of the flame. This is illustrated by analyzing the swirled burner 
dynamics considered by Palies et al. (2011a). It is shown that a relatively simple procedure including the nonlinear flame response provides estimates of the amplitudes of limit cycles while accounting for the 3D geometry of the system.

This paper is organized as follows: the swirled combustor under study is presented in conjunction with its idealized geometrical representation in section 2. Subsequently, the acoustic modeling of the combustor is explained and the flame describing function is introduced in section 3. The fourth section deals with the Helmholtz solver and numerical issues are discussed. The method used to insert the FDF into the Helmholtz computations is explained in the fifth section. After a validation of the numerical procedure, linear and nonlinear stability analyses of the target configuration is carried out in the last section. It is shown that it is possible to predict unstable modes of a swirled combustor when some information about the dissipation of the system is available. Finally, the growth rate trajectories of the fundamental mode of the combustor are calculated and compared to analytical results. These trajectories are then used to assess limit cycle amplitudes.

\section{The swirled combustor}

The experimental combustor designed and built at EM2C laboratory has been thorougly used to study the dynamics of turbulent swirled flames (Palies et al., 2009, 2010, 2011a,b,c). It comprises an upstream manifold of variable length, an axisymmetric convergent duct and a cylindrical combustion chamber of variable size schematically represented in Fig. 1. A mixture of methane and air is injected at the bottom of the upstream manifold. This mixture passes through a fine honeycomb grid structure which serves to break down large scale fluctuations. The flow then reaches a convergent duct that accelerates the stream and causes a decrease of its boundary layer thickness. As a result, the flow velocity develops a nearly flat profile just upstream of an axial swirling vane. The vane blades are twisted to induce a swirled flow characterized by a swirl number $S=0.55$ where $S$ is the ratio of the axial

flux of the tangential momentum to the product of the axial momentum flux and the injection tube radius. As shown in Fig. 1, the swirler also comprises a central rod which contributes to the flame anchoring. Under normal operating conditions, the flame is stabilized at both the central rod and at the outer lip of the injection channel. 


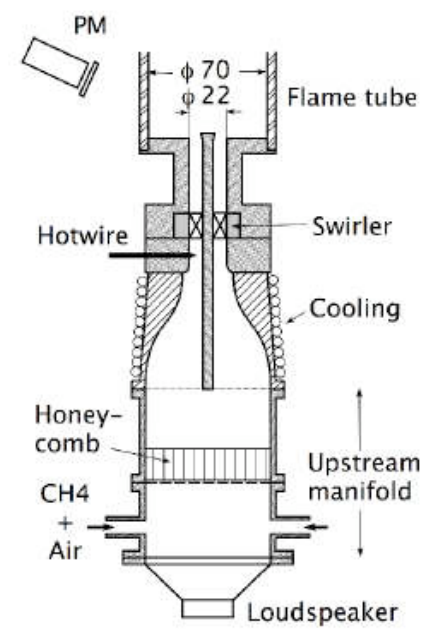

Figure 1: Swirled flow configuration examined by Palies et al. (2011a).

The stability of this system has been analyzed thanks to a nonlinear dispersion relation (Palies et al., 2011a). It is assumed that the system can be represented by three one dimensional coupled cavities (see Fig. 2). The lengths of the upstream manifold $l_{1}=96,160,224 \mathrm{~mm}$ and the convergent axisymmetrical duct $l_{2}=145 \mathrm{~mm}$ are replaced by equivalent lengths $l_{1}=$ $124.8,188.8,252.8 \mathrm{~mm}$ and $l_{2}=116.7 \mathrm{~mm}$ in the idealized geometry presented in Fig. 2 as explained in Palies et al. (2011a). Four different lengths are considered for the combustion chamber $l_{3}=100,150,200,400 \mathrm{~mm}$. An end correction of $28 \mathrm{~mm}$, which corresponds to 0.4 times the combustion chamber diameter $\left(0.4 d_{3}\right)$, is added to these values when performing analytical and numerical computations. The real and idealized geometries of the combustor are displayed in Fig. 2, where the respective transversal section dimensions are shown. The twelve different set-ups studied are shown in Tab. 1.

\section{Acoustic modeling of the swirled combustor}

\subsection{Passive flame}

The acoustic response of the idealized configuration in Fig. 2 is first examined in the absence of unsteady combustion. At the inlet of the configuration, a zero velocity fluctuation is imposed as boundary condition whereas at the outlet a zero pressure fluctuation is prescribed. A system of equations is formed by assuming that at each interface the acoustic pressure and flow rate 


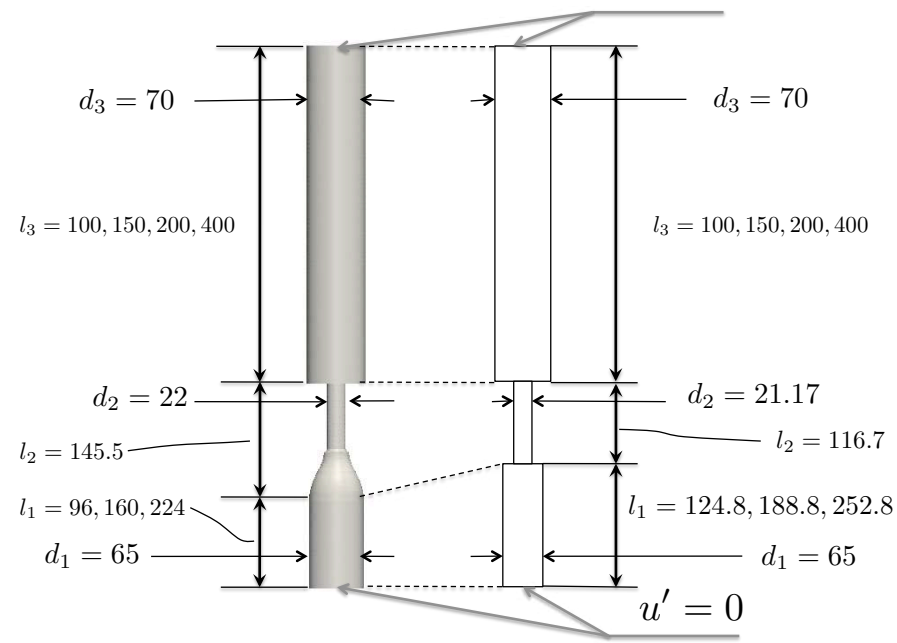

Figure 2: Swirled flow configuration explored in this study. Left: Numerical/Experimental configuration. Right: Idealized configuration adopted from Palies et al. (2011a). Dimension are given in millimeters.

Table 1: Twelve different configurations explored. Numerical computations are carried out by considering the actual dimensions of the combustor. Analytical predictions are performed on an equivalent idealized configuration. $l_{1}$ indicates the upstream manifold length and $l_{3}$ corresponds to the combustion chamber length. Dimensions are given in millimeters.

\begin{tabular}{|c|c|c|c|c|c|}
\hline \multicolumn{2}{|c|}{ Cases Studied } & $l_{3}=100$ & $l_{3}=150$ & $l_{3}=200$ & $l_{3}=400$ \\
\hline Expe./ Num. & $\boldsymbol{l}_{1}=96.0$ & \multirow{2}{*}{$\mathrm{C} 01$} & \multirow{2}{*}{$\mathrm{C} 02$} & \multirow{2}{*}{ C03 } & \multirow{2}{*}{$\mathrm{C} 04$} \\
\hline Analytics & $\boldsymbol{l}_{\mathbf{1}}=124.8$ & & & & \\
\hline Expe./ Num. & $\boldsymbol{l}_{1}=160.0$ & \multirow{2}{*}{$\mathrm{C} 05$} & \multirow{2}{*}{$\mathrm{C} 06$} & \multirow{2}{*}{$\mathrm{C} 07$} & \multirow{2}{*}{$\mathrm{C} 08$} \\
\hline Analytics & $\boldsymbol{l}_{\mathbf{1}}=188.8$ & & & & \\
\hline Expe./ Num. & $l_{1}=224.0$ & \multirow{2}{*}{ C09 } & \multirow{2}{*}{$\mathrm{C} 10$} & \multirow{2}{*}{ C11 } & \multirow{2}{*}{$\mathrm{C} 12$} \\
\hline Analytics & $\boldsymbol{l}_{\mathbf{1}}=252.8$ & & & & \\
\hline
\end{tabular}


fluctuations are continuous and by setting the respective acoustic boundary conditions. It yields:

$$
\begin{aligned}
\left.\hat{u}_{1}\right|_{x=0} & =0 \\
\left.\hat{p}_{1}\right|_{x=l_{1}} & =\left.\hat{p}_{2}\right|_{x=l_{1}} \\
\left.S_{1} \hat{u}_{1}\right|_{x=l_{1}} & =\left.S_{2} \hat{u}_{2}\right|_{x=l_{1}} \\
\left.\hat{p}_{2}\right|_{x=l_{1}+l_{2}} & =\left.\hat{p}_{3}\right|_{x=l_{1}+l_{2}} \\
\left.S_{2} \hat{u}_{2}\right|_{x=l_{1}+l_{2}} & =\left.S_{3} \hat{u}_{3}\right|_{x=l_{1}+l_{2}} \\
\left.\hat{p}_{1}\right|_{x=l_{1}+l_{2}+l_{3}^{*}} & =0
\end{aligned}
$$

where $S_{i}=\pi d_{i}^{2} / 4$ stands for the cross section area of diameter $d_{i}$ with $i=1,2$ or 3 , and $l_{3}^{*}=l_{3}+0.4 d_{3}$. In each element the pressure $\hat{p}$ and velocity $\hat{u}$ fluctuations correspond to waves propagating in the positive and negative directions:

$$
\begin{aligned}
\hat{p} & =A^{+} e^{i k x}+A^{-} e^{-i k x} \\
\bar{\rho} \bar{c} \hat{u} & =A^{+} e^{i k x}-A^{-} e^{-i k x}
\end{aligned}
$$

where the complex amplitudes $A^{+}$and $A^{-}$differ in each duct section. The resulting $6 \times 6$ linear system reads:

$$
\underbrace{\left[\begin{array}{cccccc}
1 & -1 & 0 & 0 & 0 & 0 \\
e^{i k_{u} l_{1}} & e^{-i k_{u} l_{1}} & -1 & -1 & 0 & 0 \\
\frac{S_{1}}{S_{2}} e^{i k_{u} l_{1}} & -\frac{S_{1}}{S_{2}} e^{-i k_{u} l_{1}} & -1 & 1 & 0 & 0 \\
0 & 0 & e^{i k_{u} l_{2}} & e^{-i k_{u} l_{2}} & -1 & -1 \\
0 & 0 & \Xi e^{i k_{u} l_{2}} & -\Xi e^{-i k_{u} l_{2}} & -1 & 1 \\
0 & 0 & 0 & 0 & e^{i k_{b} l_{3}^{*}} & e^{-i k_{b} l_{3}^{*}}
\end{array}\right]}_{M}\left[\begin{array}{c}
A_{1}^{+} \\
A_{1}^{-} \\
A_{2}^{+} \\
A_{2}^{-} \\
A_{3}^{+} \\
A_{3}^{-}
\end{array}\right]=\left[\begin{array}{c}
0 \\
0 \\
0 \\
0 \\
0 \\
0
\end{array}\right]
$$

where $k_{u}$ and $k_{b}$ are the wave numbers in the unburned and burned gases respectively, and

$$
\Xi=\left(S_{2} / S_{3}\right)\left(\rho_{b} c_{b}\right) /\left(\rho_{u} c_{u}\right)
$$

is a dimensionless parameter which quantifies the degree of coupling between the combustor and the injector. Modes of the three coupled cavities 
correspond to non trivial solutions of $\operatorname{det} M=0$. The following dispersion relation can be found analytically (Schuller et al., 2012):

$$
\begin{array}{r}
\cos \left(k_{b} l_{3}^{*}\right)\left[\cos \left(k_{u} l_{1}\right) \cos \left(k_{u} l_{2}\right)-\frac{S_{1}}{S_{2}} \sin \left(k_{u} l_{1}\right) \sin \left(k_{u} l_{2}\right)\right] \\
-\sin \left(k_{b} l_{3}^{*}\right) \Xi\left[\cos \left(k_{u} l_{1}\right) \sin \left(k_{u} l_{2}\right)+\frac{S_{1}}{S_{2}} \sin \left(k_{u} l_{1}\right) \cos \left(k_{u} l_{2}\right)\right]=0
\end{array}
$$

In the absence of unsteady combustion and damping, these modes are marginally stable meaning that the corresponding angular frequencies take only real values.

\subsection{Flame Describing Function}

The interaction between combustion and acoustics is modeled by making use of a FDF determined experimentally (Palies et al., 2010). The data are expressed in terms of a gain $G$ and a phase $\phi$ as follows:

$$
\mathcal{F}\left(|\hat{u}|, \omega_{r}\right)=G\left(|\hat{u}|, \omega_{r}\right) e^{i \phi\left(|\hat{u}|, \omega_{r}\right)}=\frac{\hat{\dot{Q}}\left(|\hat{u}|, \omega_{r}\right) / \overline{\dot{Q}}}{\hat{u} / \bar{u}_{b}}
$$

where $\omega_{r}$ stands for the real part of the complex angular frequency $\omega$. Two operating points at the same equivalence ratio 0.7 are considered corresponding to mean flow velocities in the injector $\bar{u}_{b}=2.67 \mathrm{~m} \cdot \mathrm{s}^{-1}$ (Case A) and $\bar{u}_{b}=4.16 \mathrm{~m} \cdot \mathrm{s}^{-1}$ (Case B) and total flame power $\overline{\dot{Q}}=1.94 \mathrm{~kW}$ (Case A) and $\overline{\dot{Q}}=3.03 \mathrm{~kW}$ (Case B) respectively. Figure 3 shows the values of the gain $G$ and phase $\phi$ for six different amplitudes of acoustic perturbations for these

two cases. Note that this is a global FDF in the sense that $\hat{\dot{Q}}$ stands for the volume integrated heat release rate fluctuations.

The FDF description of a flame can be viewed as a set of transfer functions for amplitudes of modulations which cover the linear $\left(|\hat{u}| / \bar{u}_{b}<0.1\right.$, say) as well as the nonlinear regime up to reaching $|\hat{u}| \approx \bar{u}_{b}$. In the work of Palies et al. (2010), six different ratios of $|\hat{u}| / \bar{u}_{b}$ were considered as displayed in Fig. 3. Overall, it can be stated that the different FTF present a similar behavior in both gain and phase. The gain of these FTF is characterized by two maxima with a strong local minimum in between. Here, for the smallest values of $|\hat{u}| / \bar{u}_{b}$, these maxima are observed in the vicinity of $25 \mathrm{~Hz}$ and $100 \mathrm{~Hz}$ for the Flame A, and around $50 \mathrm{~Hz}$ and $125 \mathrm{~Hz}$ for Flame B. The local minimum, on the other hand, is seen at $60 \mathrm{~Hz}$ for flame $\mathrm{A}$ and 

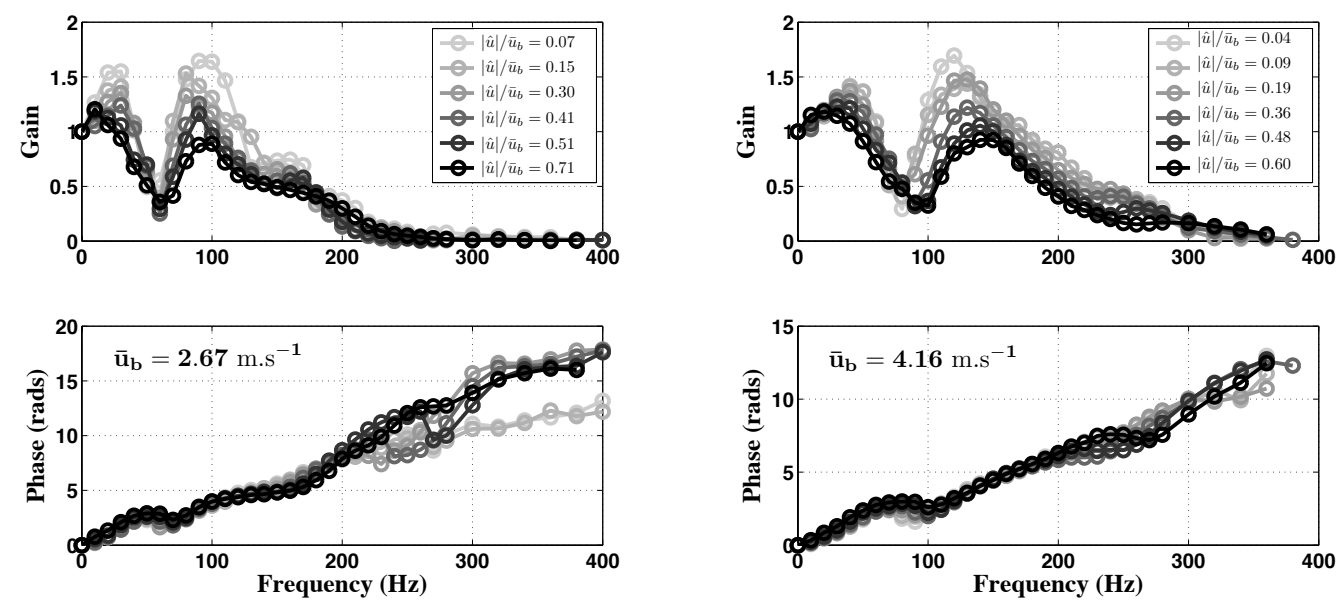

a) Flame $\mathrm{A}$

b) Flame B

Figure 3: Flame Describing Function from Palies et al. (2010)

at $98 \mathrm{~Hz}$ for flame B. It was shown by Palies et al. (2010, 2011c) that these minimum and maximum are associated to interfering acoustic and vorticity waves featuring a different propagation velocity between the plenum and the flame (perturbations in flow rate propagate at the speed of sound but also generate perturbations in the swirl number which are convected by the mean flow). When the ratio $|\hat{u}| / \bar{u}_{b}$ increases, the gain decreases and the two maxima slightly shift to higher or lower frequencies. For the highest levels of $|\hat{u}| / \bar{u}_{b}$ and for frequencies larger than $40 \mathrm{~Hz}$ one observes that the flame does not act anymore as an amplifier and that the gain is less than one. At high frequencies the gain reduces progressively to zero and the flame response is limited to frequencies lower than $250 \mathrm{~Hz}$ for flame A and $350 \mathrm{~Hz}$ for flame B. Considering the phase of these FTFs, it is observed that all curves collapse for frequencies lower than $200 \mathrm{~Hz}$. The linear behavior of the phase $\phi=\omega \tau$ implies that the time delay $\tau$ taken by the incident perturbation to reach the reactive region is roughly constant for all frequencies. In Palies et al. (2010) it is shown that $\tau$ is associated to the mean convection velocity $\bar{u}_{b}$ of the flow at the injector. This FDF can now be used to analyze the system dynamics with usteady combustion. 


\subsection{Flame acoustics coupling}

When the flame generates unsteady heat release rate fluctuations, the continuity of the acoustic flowrate Eq. (7) used in section 3.1 must be replaced by the following jump condition

$$
\left.\mathcal{K} S_{2} \hat{u}_{2}\right|_{x=l_{1}+l_{2}}=\left.S_{3} \hat{u}_{3}\right|_{x=l_{1}+l_{2}}
$$

where $\mathcal{K}$ is given by

$$
\mathcal{K}\left(\omega,\left|\hat{u}_{2}\right|\right)=\left[1+G e^{i \phi}\left(\frac{T_{3}}{T_{2}}-1\right)\right]
$$

$T_{2}$ and $T_{3}$ being the flow temperature in the second and third cavities (see Fig. 2). The resulting matrix is the same as in Eq. (11) except for the fifth row:

$$
M=\left[\begin{array}{cccccc}
1 & -1 & 0 & 0 & 0 & 0 \\
e^{i k_{u} l_{1}} & e^{-i k_{u} l_{1}} & -1 & -1 & 0 & 0 \\
\frac{S_{1}}{S_{2}} e^{i k_{u} l_{1}} & -\frac{S_{1}}{S_{2}} e^{-i k_{u} l_{1}} & -1 & 1 & 0 & 0 \\
0 & 0 & e^{i k_{u} l_{2}} & e^{-i k_{u} l_{2}} & -1 & -1 \\
0 & 0 & \mathcal{K} \Xi e^{i k_{u} l_{2}} & -\mathcal{K} \Xi e^{-i k_{u} l_{2}} & -1 & 1 \\
0 & 0 & 0 & 0 & e^{i k_{b} l_{3}^{*}} & e^{-i k_{b} l_{3}^{*}}
\end{array}\right]
$$

Modes of the three coupled cavities correspond to non trivial solutions of $\operatorname{det} M=0$. The corresponding roots are associated to complex values of $\omega$ where the real and imaginary components denote their angular oscillation frequency and growth rate respectively. Solutions with positive growth rate indicate unstable modes, while negative values correspond to damped or stable modes.

\section{The Helmholtz solver}

The Helmholtz solver, called AVSP (Nicoud et al., 2007), uses a finite volume formulation with a cell-vertex discretization on tetrahedral elements. AVSP solves the eigenvalue problem defined by the homogeneous Helmholtz equation. For a passive flame, this equation reads :

$$
\nabla \cdot\left(\bar{c}^{2} \nabla \hat{p}\right)+\omega^{2} \hat{p}=0
$$


in which $\bar{c}$ and $\hat{p}$ stand for the mean sound velocity and the acoustic pressure fields respectively. Solutions of this equation subject to specific boundary conditions yield the acoustic modes (complex amplitude $\hat{p}$ ) and eigenfrequencies (the complex valued angular frequency $\omega$ ). This equation accounts for spatial gradients in the mean sound velocity $\bar{c}$ induced by the presence of a flame. The Helmholtz equation Eq. (18) only holds for low Mach number flows, which is generally the case in turbine combustion chambers. Standard boundary conditions are of the Neumann type $(\nabla \hat{p} \cdot \boldsymbol{n}=0$, where $\boldsymbol{n}$ is the unit normal vector to the boundary, pointing outwards) or of the Dirichlet type $(\hat{p}=0)$. While the former is applied on a rigid wall, the latter is suitable for pressure outlets discharging in the atmosphere. In order to account for nonlinear acoustic-flame interactions, it is necessary to introduce a local flame describing function which relates the local value of the unsteady heat release rate $\hat{\dot{q}}(\boldsymbol{x})$ to the acoustic velocity in a reference section and reference direction $\hat{\boldsymbol{u}}_{\text {ref }} \cdot \mathbf{n}_{\text {ref }}$ :

$$
\mathcal{F}_{\mathrm{loc}}\left(\boldsymbol{x}, \omega_{r},|\hat{u}|\right)=G_{\mathrm{loc}}\left(\boldsymbol{x}, \omega_{r},|\hat{u}|\right) e^{i \phi_{\mathrm{loc}}\left(\boldsymbol{x}, \omega_{r},|\hat{u}|\right)}=\frac{\hat{\dot{q}}(\boldsymbol{x}) / \overline{\dot{q}}_{\mathrm{ref}}}{\hat{\boldsymbol{u}}_{\mathrm{ref}} \cdot \mathbf{n}_{\mathrm{ref}} / \bar{u}_{\mathrm{ref}}}
$$

where $G_{\text {loc }}$ and $\phi_{\text {loc }}$ represent the local gain and the local phase lag respectively. The quantities $\bar{u}_{\text {ref }}$ and $\overline{\dot{q}}_{\text {ref }}$ stand for the reference velocity and heat release rate respectively, introduced for units consistency. The Helmholtz equation with a heat release rate source $\hat{\dot{q}}$ is given for example by (Poinsot and Veynante, 2005):

$$
\nabla \cdot\left(\bar{c}^{2} \nabla \hat{p}\right)+\omega^{2} \hat{p}=\boldsymbol{i} \omega(\gamma-1) \hat{\dot{q}}(\boldsymbol{x})
$$

Equation (20) is now combined with Eq. (19) to give:

$$
\left.\nabla \cdot\left(\bar{c}^{2} \nabla \hat{p}\right)+\omega^{2} \hat{p}=\boldsymbol{i} \omega(\gamma-1) \frac{\overline{\dot{q}} \hat{\boldsymbol{u}}_{\mathrm{ref}} \cdot \mathbf{n}_{\mathrm{ref}}}{\bar{u}_{\mathrm{ref}}} G_{\mathrm{loc}}\left(\boldsymbol{x}, \omega_{r},|\hat{u}|\right) e^{i \phi_{\mathrm{loc}}\left(\boldsymbol{x}, \omega_{r},|\hat{u}|\right.}\right)
$$

Using the linearized momentum equation for low Mach number flows $(\boldsymbol{i} \omega \bar{\rho} \hat{\boldsymbol{u}}=\nabla \hat{p})$, Eq. (21) becomes:

$$
\underbrace{\nabla \cdot\left(\bar{c}^{2} \nabla \hat{p}\right)}_{\mathcal{A} \hat{\mathbf{p}}}+\underbrace{\omega^{2} \hat{p}}_{\omega^{2} \hat{\mathbf{p}}}-\underbrace{(\gamma-1) \frac{\overline{\dot{q}} \nabla \hat{p}_{\mathrm{ref}} \cdot \mathbf{n}_{\mathrm{ref}}}{\bar{\rho} \bar{u}_{\mathrm{ref}}} G_{\mathrm{loc}}\left(\boldsymbol{x}, \omega_{r},|\hat{u}|\right) e^{i \phi_{\mathrm{loc}}\left(\boldsymbol{x}, \omega_{r},|\hat{u}|\right)}}_{\mathcal{H}\left(\hat{\mathbf{p}}_{\mathrm{ref}}, \omega\right)}=0
$$




$$
\mathcal{A} \hat{\mathbf{p}}+\omega^{2} \hat{\mathbf{p}}+\mathcal{H}\left(\hat{\mathbf{p}}_{\text {ref }}, \omega\right)=0
$$

Here $\hat{\mathbf{p}}$ represents the eigenvectors of the system and is associated to the discretized acoustic field. The complex valued scalar $\omega$ represents the eigenvalues (eigenfrequencies) of the system. The matrix $\mathcal{A}$ that comes up once the operator $\nabla \cdot\left(\bar{c}^{2} \nabla\right)$ is discretized depends on the discretization scheme. Finally, $\mathcal{H}$ is a complex vector that depends, in addition to $\hat{\mathbf{p}}_{\text {ref }}$, on the eigenvalue $\omega$, the gain $G_{\text {loc }}$ and the phase lag $\phi_{\text {loc }}$, and the mean field. This mathematical problem reduces to $\mathcal{A} \hat{\mathbf{p}}+\omega^{2} \hat{\mathbf{p}}=0$ when the flame is neutral $(\mathcal{H}=0)$. This is a linear eigenvalue problem which is solved in this study by means of the Arnoldi Algorithm (Trefethen and Bau, 1997).

When the third term of Eq. (23) is taken into account (the flame produces heat release rate fluctuations), the problem becomes a nonlinear eigenvalue problem and an additional algorithm is needed to obtain a linearized version which can be used in an iterative procedure. Here, the flame term is given at a frequency value $\omega^{j}=\omega_{r}^{j}+i \omega_{i}^{j}$ for the $j$-th iteration and Eq. (24) is used to calculate an updated value $\omega^{j *}$ of $\omega$ :

$$
\underbrace{\left[\nabla \cdot \bar{c}^{2} \nabla-(\gamma-1) \frac{\overline{\dot{q}}}{\bar{\rho} \bar{u}_{\mathrm{ref}}} G_{\mathrm{loc}}\left(\boldsymbol{x}, \omega_{r}^{j},|\hat{u}|\right) e^{i \phi_{\mathrm{loc}}\left(\boldsymbol{x}, \omega_{r}^{j},|\hat{u}|\right)} \nabla_{\mathrm{ref}}\right]}_{\mathcal{A}^{*} \hat{\mathbf{p}}} \hat{p}+\underbrace{\left(\omega^{j *}\right)^{2} \hat{p}}_{\omega^{2} \hat{\mathbf{p}}}=0
$$

In this expression, the operator $\nabla_{\text {ref }}$ should be understood as the gradient at the reference point and along the reference direction $\mathbf{n}_{\text {ref. }}$. Solving Eq. (24) provides, in addition to the respective eigenvectors $\hat{\mathbf{p}}$, the eigenvalues $\omega$ of the problem. The objective is now to find a suitable iterative procedure in order to make $\omega^{j} \rightarrow \omega$. The simplest but at the same time most robust technique consists in applying the fixed point algorithm with relaxation. Naming the entire system as $g$, this algorithm can be summarized by the following relation

$$
\omega^{j+1}=\alpha \omega^{j *}+(1-\alpha) \omega^{j} \quad \text { where } \quad g: \omega^{j} \mapsto \omega^{j *}
$$

In this expression, $0 \leq \alpha \leq 1$ denotes the relaxation coefficient of the method. Variables of Eq. (25) are summarized in table 2. Note that when $\alpha=1$, this algorithm reduces to the standard fixed point method where $\omega^{j+1}=$ $g\left(\omega^{j}\right)$. The iteration procedure stops once $\omega^{j} \simeq \omega^{j+1}$. Assuring convergence is a difficult task when the standard fixed point algorithm is applied to some 
Table 2: Variables for the fixed point algorithm with relaxation

\begin{tabular}{ccc}
\hline$\omega^{j}$ & $\rightarrow$ & Input of computation $j$ \\
$\omega^{j *}$ & $\rightarrow$ & Output of computation $j$ \\
$\omega^{j+1}$ & $\rightarrow$ & Input of computation $j+1$ \\
$\alpha$ & $\rightarrow$ & Relax coefficient taken as 0.5 in this study. \\
\hline
\end{tabular}

Table 3: Mean thermodynamical parameters

\begin{tabular}{|c||c|c|c|}
\hline & $\bar{T}(\mathrm{~K})$ & $\bar{p}(\mathrm{~Pa})$ & $\gamma$ \\
\hline \hline Upstream of the flame & 300 & 101325 & 1.4 \\
\hline Downstream of the flame & 1600 & 101325 & 1.4 \\
\hline
\end{tabular}

of the cases studied. However, in the present study it was observed that when relaxation with $\alpha=0.5$ is added to the standard algorithm convergence is always achieved. Moreover, only a small number of iterations $\approx 10)$ is required to reach a suitable value of $\epsilon=\left|\omega^{j}-\omega^{j *}\right|<0.01$, when the initial guess $\omega^{0}$ is taken as the solution of the passive flame problem (Eq. (18)).

\section{Helmholtz solver computations}

The AVSP Helmholtz solver is used in a first step to determine the eigenvalues of Eq. (21). The mean sound velocity $\bar{c}$ defines the effect of the steady flame and this distribution must be available at each point of the geometry. In addition to $\bar{c}$, a local gain $G_{\text {loc }}$ and a local phase $\phi_{\text {loc }}$ must be assigned which, as shown later in this section, must be consistent with the measurements of the FDF described in section 3.2.

The field of $\bar{c}$ can, for example, be deduced from numerical simulations. It is here deduced from an approximate flame shape modeled following a pattern given by flame images (Fig. 4) provided in Palies et al. (2010). By following this geometrical pattern, it is possible to establish a mean sound velocity field (Fig. 5(a)). The thermodynamical values shown in Tab. 3 are implicitly accounted for. It has been checked, but not shown in this article, that results (growth rate and eigenfrequencies) do not depend on the details of the flame shape, in agreement with the fact that the flame is acoustically compact $\left(\mathrm{h}_{\text {flame }} / \lambda_{\mathrm{ac}} \approx 0.05\right.$ where $\mathrm{h}_{\text {flame }}$ and $\lambda_{\mathrm{ac}}$ represent the height of the flame and the smallest acoustic wavelength considered respectively).

The values of the gain $G$ and phase $\phi$ provided by experiments are global values, relevant to the volume integrated response $\hat{\dot{Q}}$ of the flame. The 


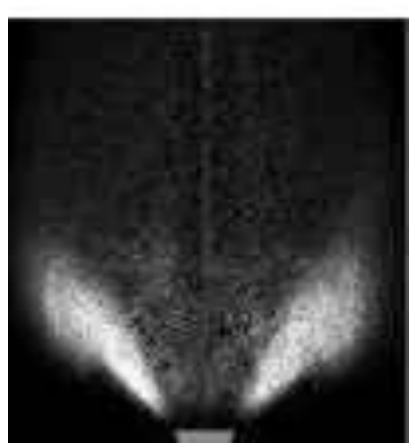

a) Flame $A$

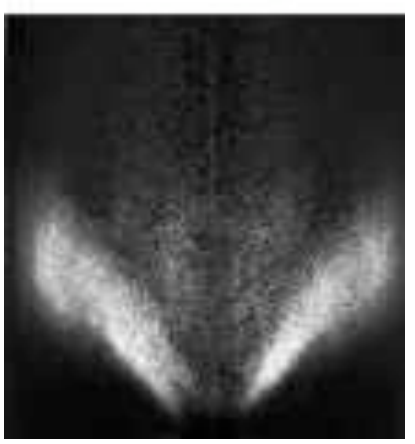

b) Flame B

Figure 4: Trace of the flame chemiluminescence in the symmetry plane of the burner. (Palies et al., 2010)

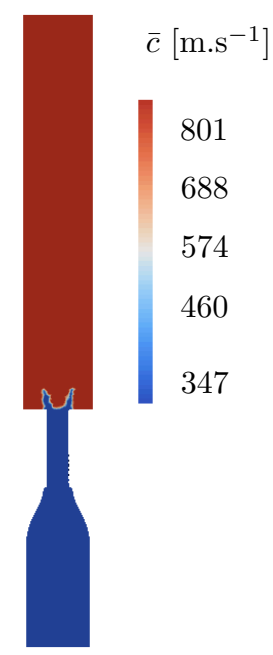

(a)

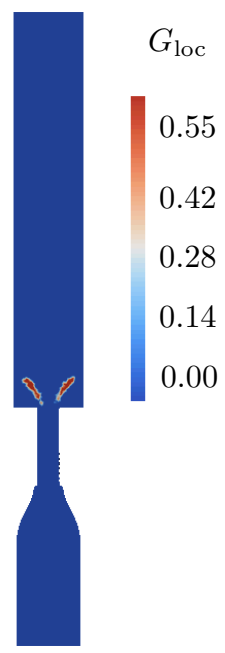

(b)

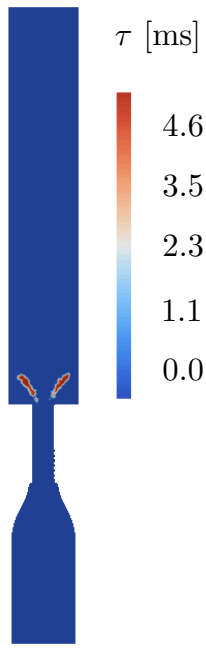

(c)

Figure 5: Example of three fields needed for AVSP computations (Flame A). (a) Mean sound velocity field $\left(\mathrm{m} . \mathrm{s}^{-1}\right)$, (b) Example of $G_{l o c}$ field or $\omega_{r}=2 \pi 60 \mathrm{rad} \mathrm{s}^{-1}$ and $|\hat{u}| / \bar{u}_{b}=0.07$, (c) Example of field for the time lag $\tau=\phi_{l o c} / \omega_{r}$ for $\omega_{r}=2 \pi 60 \mathrm{rad} \mathrm{s}^{-1}$ and $|\hat{u}| / \bar{u}_{b}=0.07$. 
Helmholtz solver requires local values of these parameters. From Eq. (19) the local unsteady heat release rate $\hat{\dot{q}}$ can be then expressed as:

$$
\hat{\dot{q}}(\boldsymbol{x})=\hat{\boldsymbol{u}}_{\mathrm{ref}} \cdot \mathbf{n}_{\mathrm{ref}} / \bar{u}_{\mathrm{ref}} G_{l o c}\left(\boldsymbol{x}, \omega_{r},|\hat{u}|\right) e^{i \phi_{l o c}\left(\boldsymbol{x}, \omega_{r},|\hat{u}|\right)} \overline{\dot{q}}_{\mathrm{ref}}
$$

Integrating Eq. (26) over the entire volume of the combustor leads to:

$$
\int_{V} \hat{\dot{q}}(\boldsymbol{x}) d V=\int_{V} \hat{\boldsymbol{u}}_{\mathrm{ref}} \cdot \mathbf{n}_{\mathrm{ref}} / \bar{u}_{\mathrm{ref}} G_{l o c}\left(\boldsymbol{x}, \omega_{r},|\hat{u}|\right) e^{i \phi_{l o c}\left(\boldsymbol{x}, \omega_{r},|\hat{u}|\right)} \overline{\dot{q}}_{\mathrm{ref}} d V
$$

Noting that $\hat{\boldsymbol{u}}_{\text {ref }} \cdot \mathbf{n}_{\text {ref }}$ and $\bar{u}_{\text {ref }}$ are values independent of $\boldsymbol{x}$, considering the flame as compact (the gain $G_{\text {loc }}$ and the phase $\phi_{\text {loc }}$ being uniform in the flame zone and set to zero elsewhere) Eq. (27) becomes:

$$
\int_{V} \hat{\dot{q}}(\boldsymbol{x}) d V=\hat{\boldsymbol{u}}_{\mathrm{ref}} \cdot \mathbf{n}_{\mathrm{ref}} / \bar{u}_{\mathrm{ref}} G_{l o c}\left(\boldsymbol{x}, \omega_{r},|\hat{u}|\right) e^{i \phi_{l o c}\left(\boldsymbol{x}, \omega_{r},|\hat{u}|\right)} \overline{\dot{q}}_{\mathrm{ref}} V_{f}
$$

where $V_{f}$ is the volume of the compact flame. The local flame description will be consistent with the global flame response if

$$
\int_{V} \hat{\dot{q}}(\boldsymbol{x}) d V=\hat{\dot{Q}}
$$

Combining Eq. (28) and Eq. (14), choosing $\bar{u}_{\text {ref }}=\bar{u}_{b}$ and $\phi_{\text {loc }}=\phi$ leads to:

$$
\overline{\dot{Q}} G\left(\omega_{r},|\hat{u}|\right)=G_{\text {loc }} \overline{\dot{q}}_{\text {ref }} V_{f}
$$

It was checked that if the constraint Eq. (30) is satisfied, results are independent of the details of the definition of the flame region. Should the latter be made smaller, the flame volume decreases and the local response $G_{\text {loc }}$ increases because of Eq. (30). In the end, the overall flame response is recovered. By choosing $\overline{\dot{q}}_{\text {ref }}=\overline{\dot{Q}} / V_{f}$, the local value of the gain $G_{\text {loc }}$ is then given by:

$$
G_{\text {loc }}=G\left(\omega_{r},|\hat{u}|\right)
$$

within the flame, while it is zero outside the flame. In the same way, the local value of the phase is chosen as:

$$
\phi_{\text {loc }}=\phi\left(\omega_{r},|\hat{u}|\right)
$$

inside the flame and $\phi_{\text {loc }}=0$ outside. Two examples of local gain and phase lag fields are shown in Figs. 5(b,c). The two operating points correspond to thermal powers $\overline{\dot{Q}}=1.94 \mathrm{~kW}$ and $\overline{\dot{Q}}=3.03 \mathrm{~kW}$. 
Table 4: Three types of computations.

\begin{tabular}{|c||c|c|}
\hline Type & Geometry & Solver \\
\hline \hline T1 & Idealized configuration (1D by parts) & Analytics \\
\hline T2 & Idealized configuration (1D by parts) & Helmholtz solver \\
\hline T3 & Real configuration (3D) & Helmholtz solver \\
\hline
\end{tabular}

\section{Results}

\subsection{Numerical and analytical computations on a simplified configuration}

It is first natural to validate the computational tool by considering an idealized geometry and comparing analytical and numerical results ( $\mathrm{T} 1$ and T2 in Tab. 4). Here, the experimental data for the FDF presented in section 3.2 are not accounted for. Instead, the idealized geometry, already described in section 2 and shown in Fig. 2, is considered together with increasing values of both gain $G$ and time delay $\tau$. The analysis is carried out for the first acoustic mode of the coupled cavities system in the configuration C05 (see Tab. 1). Calculations displayed in Fig. 6 are in good agreement with analytical results.

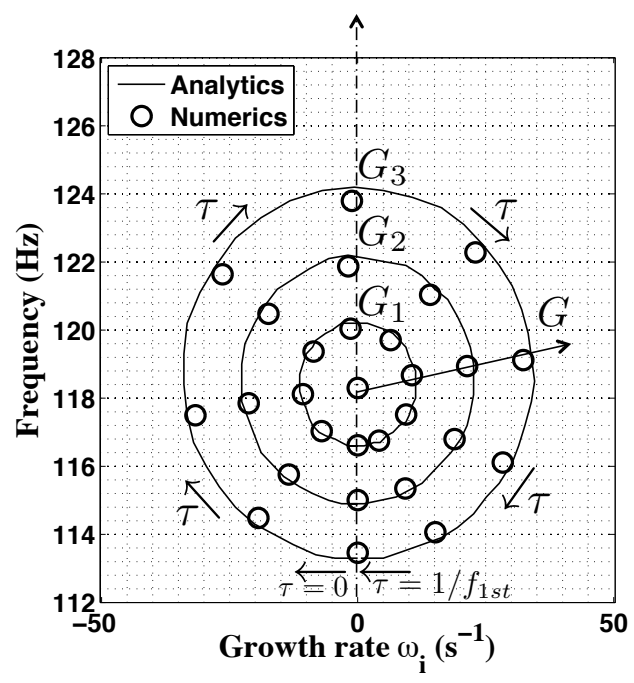

Figure 6: Analytical (T1) and numerical (T2) computations performed on the C05 configuration. The gain takes four different values: $G_{0}=0, G_{1}=0.5, G_{2}=1.0$ and $G_{3}=1.5$. The time lag $\tau$ takes values from $\tau=0$ to $\tau=1 /\left(f_{1 \text { st }}\right)$ where $f_{1 \text { st }}=118.4 \mathrm{~Hz}$. 
In addition to validation purposes of the Helmholtz solver, simulations shown in Fig. 6 also provide indications on the influence of the unsteady heat release rate $\hat{\dot{q}}$ on the eigenfrequencies of a typical combustion system. It shows how the gain $G$ and the time delay $\tau$ of a given transfer function affects the resonance frequency and the growth rate of acoustic modes in an academic $1 \mathrm{D}$ combustor. For a steady flame when $G=0$, i.e. $\hat{\dot{q}}=0$, there is only one eigenfrequency corresponding to the first acoustic mode of the system $f_{1 \text { st }}$. This eigenfrequency is the center of the circular trajectories shown in Fig. 6 and is a real number $\left(\operatorname{Im}\left(f_{1 \mathrm{st}}\right)=0\right)$. Once a gain is attributed to the transfer function $(G \neq 0)$, and $\tau$ is kept equal to zero, it is seen that the resonance frequency of the system decreases and that the growth rate remains zero. Keeping the same value of $G, \tau$ is made to change from zero to $\tau=1 /\left(2 f_{1 \mathrm{st}}\right)$. Eigenfrequencies lie on a semi-circumferential trajectory swept clockwise which is located on the left half of the complex plane. The imaginary component of the eigenfrequency, i.e. the growth rate of the acoustic mode, is always negative and the system is as a consequence stable. Meanwhile, the real part of the eigenfrequency, i.e. the resonance frequency of the acoustic mode, goes from a minimum to a maximum value. For larger values of the time lag $1 /\left(2 f_{1 \mathrm{st}}\right)<\tau<1 / f_{1 \mathrm{st}}$, the trajectories lie on the right half of the complex plane. The growth rate now takes positive values and the system is unstable. In this case, resonance frequencies change from a maximum to a minimum value. It is also observed that the radius of the circumferential trajectories in Fig. 6 only depends on the gain of the transfer function $\mathcal{F}$. To sum up, the phase $\phi=2 \pi f_{1 s t} \tau$ of $\mathcal{F}$ defines whether the combustion system is stable or not, while the gain $G$ acts on the resonance frequency shift with respect to the original value. Besides, the good agreement between the $T_{1}$ and $T_{2}$ types of calculations presented in Fig. 6 shows that the Helmholtz solver gives virtually the same results as the analytical approach when the idealized geometry is considered. As a consequence, any differences between $T_{1}$ and $T_{3}$ computations would be most probably due to geometrical effects. Such computations are considered in the next section.

\subsection{Stability Analysis}

In this section the numerical simulations T3 considering the real geometry of the combustor with active flames are discussed. Figure 7 shows the values of the growth rate $\omega_{i}$ of the first acoustic mode for the twelve configurations investigated (see Tab. 1). These growth rates $\omega_{i}$ are computed considering the smallest value of $|\hat{u}| / \bar{u}_{b}$ for the two flames regimes $\mathrm{A}$ and $\mathrm{B}$. For a system 
with no intrinsic dissipation, stability would be given directly by the sign of $\omega_{i}$. Nevertheless, for complex configurations, acoustic energy can be dissipated in various ways. Measuring this damping rate under realistic operating conditions is difficult and there is no well established experimental procedure for this measurement.

Palies et al. (2011a) evaluated the acoustic damping of their combustor by analyzing the associated resonance sharpness, which was obtained by submitting the combustor to external acoustic waves and measuring the response for a range of frequencies around a resonance. From this response one finds the frequency bandwidth $\Delta f$ which can be used to determine the quality factor $Q=f / \Delta f$. Assuming that the system behaves like a harmonic oscillator, one deduces an effective damping rate $\alpha^{\prime}=\pi \Delta f$. This method is used in many cases to obtain estimates of the damping in a complex system. It rests on the assumption that the dynamic response can be assimilated to that of a second order system, which is reasonable for acoustic systems. By making use of a projection on the modes of the system, it is possible to show that the modal amplitudes satisfy a set of second order differential equations coupled by acoustic nonlinearities and unsteady heat release source terms. It is thus natural to use a second order model to represent damping of each particular mode. There is no special assumption on the flame symmetry but one has to consider the flame contribution to the effective damping.

The measurements of $\alpha^{\prime}$ were performed for stable operating regimes chosen within configurations C01-C12. It is shown in Palies et al. (2011a) that the resonance sharpness of all stable configurations (C01, C02, C05, C06, C09 and $\mathrm{C} 10)$ is similar. As a consequence, the configuration $\mathrm{C} 05$ will be considered as representative in what follows. The values of the effective damping rate of $\mathrm{C} 05$ were found to be $\alpha^{\prime}=23 \mathrm{~s}^{-1}$ for flame A and $\alpha^{\prime}=56 \mathrm{~s}^{-1}$ for flame B. This effective damping rate $\alpha^{\prime}$ is a function of both the 'actual' damping rate of the system $\alpha$ and the damping or growth rate attributed to flame dynamics $\omega_{i}$. More precisely it is defined as $\alpha^{\prime}=\alpha-\omega_{i}$, where $\omega_{i}$ is taken as positive if the flame acts as an amplifier and as negative if the flame damps acoustic oscillations. In cases where $\omega_{i}>0$, the 'actual' damping rate of the system $\alpha$ is estimated by adding to $\alpha^{\prime}$ the damping that was removed by the growth rate induced by the flame.

In addition to $\alpha^{\prime}$, which is associated to all stable configurations, the growth rate induced by the flame $\omega_{i}$ should be also representative of all stable configurations. As a consequence, this value of the growth rate $\omega_{i}^{\text {av }}$ is then computed as the average of all independent values $\omega_{i}$ of the stable 
cases C01, C02, C05, C06, C09 and C10 for each operating condition A and B. Values equal to $\omega_{i}^{\text {av }}=59 \mathrm{~s}^{-1}$ for the Flame $\mathrm{A}$ and to $\omega_{i}^{\text {av }}=62 \mathrm{~s}^{-1}$ for the Flame $\mathrm{B}$ are obtained. The damping rate of the system for flame $\mathrm{A}$ is therefore estimated as $\alpha_{A}=82 \mathrm{~s}^{-1}$ whereas it is $\alpha_{B}=125 \mathrm{~s}^{-1}$ for flame B. In this work these values were kept for all the cases investigated with flame $\mathrm{A}$ and flame B respectively. This is different from the choice made by Palies et al. (2011a) where a unique value of $\alpha$ was defined as $\alpha \simeq 55 \pm 10 \mathrm{~s}^{-1}$.

Assuming now that all configurations present similar values of $\alpha_{A}$ and $\alpha_{B}$ for each operating condition $\mathrm{A}$ and $\mathrm{B}$ respectively, it is possible to predict if the system is either stable, marginally stable or unstable. The regime is considered here stable if the growth rate is smaller than the damping rate, marginally stable if the growth rate lies between $\alpha \pm 10 \mathrm{~s}^{-1}$ and unstable if the growth rate is larger than the damping rate values. Numerical results are shown in Fig. 7 and summarized in Tab. 5 where the actual state observed in the experiments is also shown. Numerical computations succeed in most of the cases to predict stability, marginal stability and instability of the swirled combustor. The partial disagreements reported in Fig. 7 correspond to cases where the experiments gives marginal stability ( $\mathrm{S}-\mathrm{U}$ in Tab. 5) while the computation predicts instability (U in Tab. 5) or vice versa. Note also that no large differences between predictions and measurements were observed; the overall agreement is thus very good.

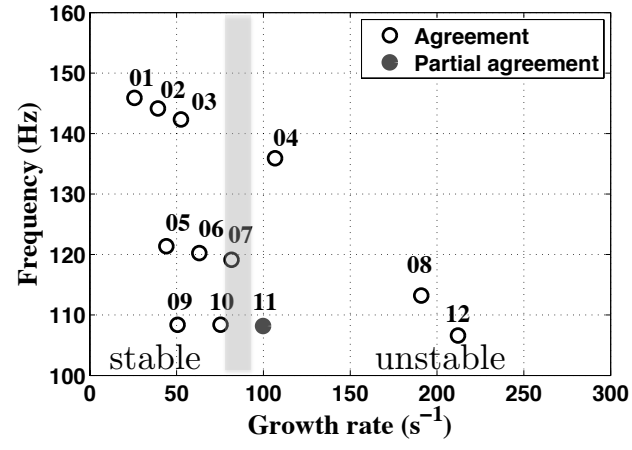

(a) Flame A

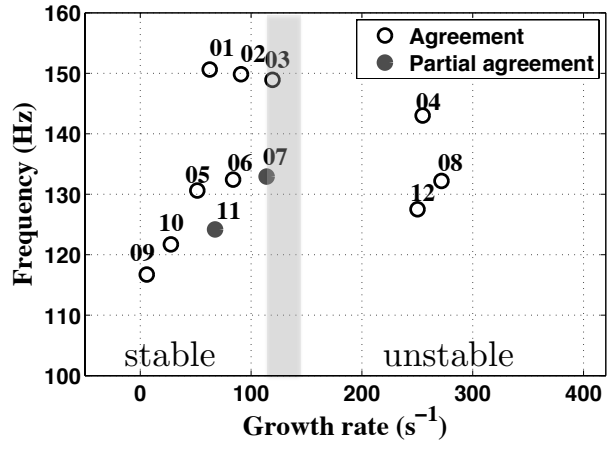

(b) Flame B

Figure 7: Linear stability prediction. The gray bounds indicate the marginally stable region defined by $\alpha \pm 10 \mathrm{~s}^{-1}$. Empty symbols indicate agreement with experimental results while filled symbols represent partial agreement (see Tab. 5). 
Table 5: Linear stability analysis of flame A and flame B. Comparison between experimental and numerical results. (S) Stable, (S-U) Marginally stable/unstable, (U) Unstable. The geometrical configurations $\mathrm{C} 01$ to $\mathrm{C} 12$ are defined in Tab. 1.

\begin{tabular}{|c|c|c|c|c|c|c|c|c|}
\hline CASE & \multicolumn{4}{|c|}{ Flame A } & \multicolumn{4}{|c|}{ Flame B } \\
\hline & $\mathrm{C} 01$ & $\mathrm{C} 02$ & $\mathrm{C} 03$ & $\mathrm{C} 04$ & $\mathrm{C} 01$ & $\mathrm{C} 02$ & $\mathrm{C} 03$ & $\mathrm{C} 04$ \\
\hline Experiment & $\mathrm{S}$ & $\mathrm{S}$ & $\mathrm{S}$ & $\mathrm{U}$ & $\mathrm{S}$ & $\mathrm{S}$ & S-U & $\mathrm{U}$ \\
\hline Simulation & $\mathrm{S}$ & $\mathrm{S}$ & $\mathrm{S}$ & $\mathrm{U}$ & $\bar{S}$ & $\mathrm{~S}$ & $\mathrm{~S}-\mathrm{U}$ & $\mathrm{U}$ \\
\hline & $\mathrm{C} 05$ & $\mathrm{C} 06$ & $\mathrm{C} 07$ & $\mathrm{C} 08$ & $\mathrm{C} 05$ & $\mathrm{C} 06$ & $\mathrm{C} 07$ & $\mathrm{C} 08$ \\
\hline Experiment & $\mathrm{S}$ & $\mathrm{S}$ & S-U & $\mathrm{U}$ & $\mathrm{S}$ & $\mathrm{S}$ & $\mathrm{S}$ & $\mathrm{U}$ \\
\hline Simulation & S & $\mathrm{S}$ & $\mathrm{S}-\mathrm{U}$ & $\mathrm{U}$ & S & $\mathrm{S}$ & $\mathrm{S}-\mathrm{U}$ & $\mathrm{U}$ \\
\hline & C09 & C10 & C11 & $\mathrm{C} 12$ & $\mathrm{C} 09$ & C10 & C11 & C12 \\
\hline Experiment & $\mathrm{S}$ & $\mathrm{S}$ & S-U & $\mathrm{U}$ & $\mathrm{S}$ & $\mathrm{S}$ & S-U & $\mathrm{U}$ \\
\hline Simulation & $\mathrm{S}$ & $\mathrm{S}$ & $\mathrm{U}$ & $\mathrm{U}$ & $\mathrm{S}$ & $\mathrm{S}$ & $\mathrm{S}$ & $\mathrm{U}$ \\
\hline
\end{tabular}

\subsection{Growth rate trajectories}

The growth rate variation of the fundamental mode is now analyzed as a function of the amplitude of the acoustic oscillations. Figures 8 and 9 show the growth rate and resonance frequency trajectories for flame A and B as a function of the perturbation level $|\hat{u}| / \bar{u}_{b}$ taking values in the range 0.01 to 0.71 . These figures indicate that for large amplitudes of acoustic velocity perturbations the growth rate decreases. A stationary state, i.e. a limit cycle, is reached when the growth rate equals the damping rate of the system. The amplitude at which this happens corresponds to the oscillation level at the limit cycle.

It is worth stressing that predictions of limit cycles are extremely sensitive to the measurements of the damping rate of the system. Uncertainties of the order of 1 to $10 \mathrm{rad} \mathrm{s}^{-1}$ may lead to differences up to $1-2 \mathrm{~m} . \mathrm{s}^{-1}$ in the prediction of the acoustic velocity corresponding to $500 \mathrm{~Pa}$ in combustion noise. As a result, one cannot expect precise estimates of oscillation levels because accurate measurements of the damping rate are not available.

Nevertheless, interesting observations can be made from growth rate and frequency trajectories. For almost all the trajectories, small acoustic perturbations $\left(|\hat{u}| / \bar{u}_{b} \approx 0.01\right)$ present the highest values of $\omega_{i}$. However, as long as $\omega_{i}<\alpha$ the system dissipates all the acoustic energy generated by the coupling with the flame. In only ten, out of twenty-four trajectories, the acoustic mode amplitude is augmented $\left(\omega_{i}>\alpha\right)$ until the acoustic growth rate equals the damping rate of the system. There is only one trajectory (C04-Flame B) 
in which $\omega_{i}$ does not equal $\alpha$ for the range of values $|\hat{u}| / \bar{u}_{b}$ displayed. However, the corresponding crossing point may be estimated by extrapolating the trajectory to larger values of $|\hat{u}| / \bar{u}_{b}$. Results of $|\hat{u}| / \bar{u}_{b}$, for which $\omega_{i} \approx \alpha$, are summarized in Tab. 6 together with measurements. Although there is a general underprediction of these values compared to experimental results, there is a good qualitative assessment of the intensity of oscillation present in the system. Numerical results, on the one hand, show that, for flame A, the strongest combustion instability takes place in $\mathrm{C} 12$, closely followed by $\mathrm{C} 08$ and $\mathrm{C} 04 ; \mathrm{C} 07$ and $\mathrm{C} 11$ are estimated to be the weakest instabilities for Flame A. On the other hand, for Flame B, simulations predict that C04 would experience the highest amplitude level followed by C08 and C12; C03 is estimated to experience a weak combustion instability in this case. All these observations correspond to similar trend to experimental data. The weakest instability for Flame B is however not well predicted by these simulations. This takes place for the $\mathrm{C} 11$ case in the experiments, while results obtained with the Helmholtz solver indicate that C07 yields the weakest unstable mode.

The predicted oscillation frequencies at limit cycles can be compared to experimental data. Evolutions of the instability frequencies are shown in Fig. 9. Nine trajectories are of special interest: C04, C07, C08, C11 and C12 for Flame A, and C03, C04, C08 and C12 for Flame B. These trajectories are intersected by the values of $|\hat{u}| / \bar{u}_{b}$ found at limit cycles providing quantitative estimates of this oscillating state. Results are shown in Tab. 6. For all cases there is a slight overestimation of this frequency ranging from $2 \mathrm{~Hz}$ for $\mathrm{C} 04-\mathrm{A}$ to $10 \mathrm{~Hz}$ for C08-B. Nevertheless, a good qualitative estimate is again obtained by the present methodology. For flame A, the highest frequency (within the measurable configurations) is predicted for the C04 case, while intermediate frequencies are found for the C07, C08 and C11 configurations, the lowest oscillation frequency corresponding to C12. For flame B, the highest frequency is found for C03 closely followed by C04; the lowest frequencies are predicted for $\mathrm{C} 08$ and C12. Again, these results are in good agreement with measurements.

\subsection{Computing growth rate trajectories for the idealized geometry}

In the previous section growth rates and instability frequencies were estimated on the actual 3D geometry by means of a Helmholtz solver. It is also interesting to examine predictions obtained with the analytical model given by Eq. (17) with the idealized configuration shown in Fig. 2. Calculations 

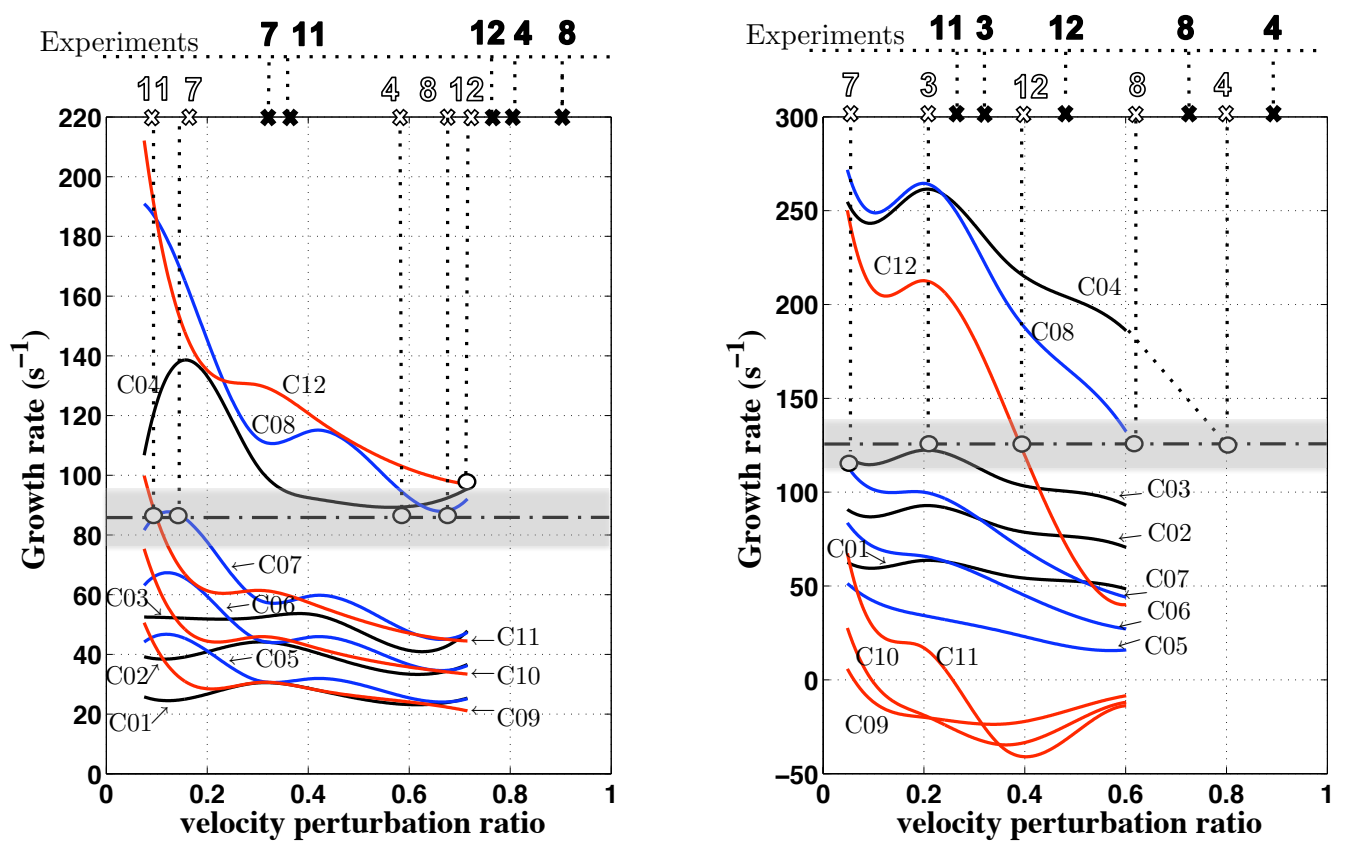

Figure 8: Growth rate trajectories as a function of the velocity fluctuation level $|\hat{u}| / \bar{u}_{b}$ at the flame base. Flame A (left) and Flame B (right). White crosses indicate predictions and black crosses correspond to measured limit cycle levels. The dashed-dotted lines surrounded by the gray band indicate the region where the growth rate is balanced by damping.

$\longrightarrow$ C01-C04, - C05-C08, — C09-C12 

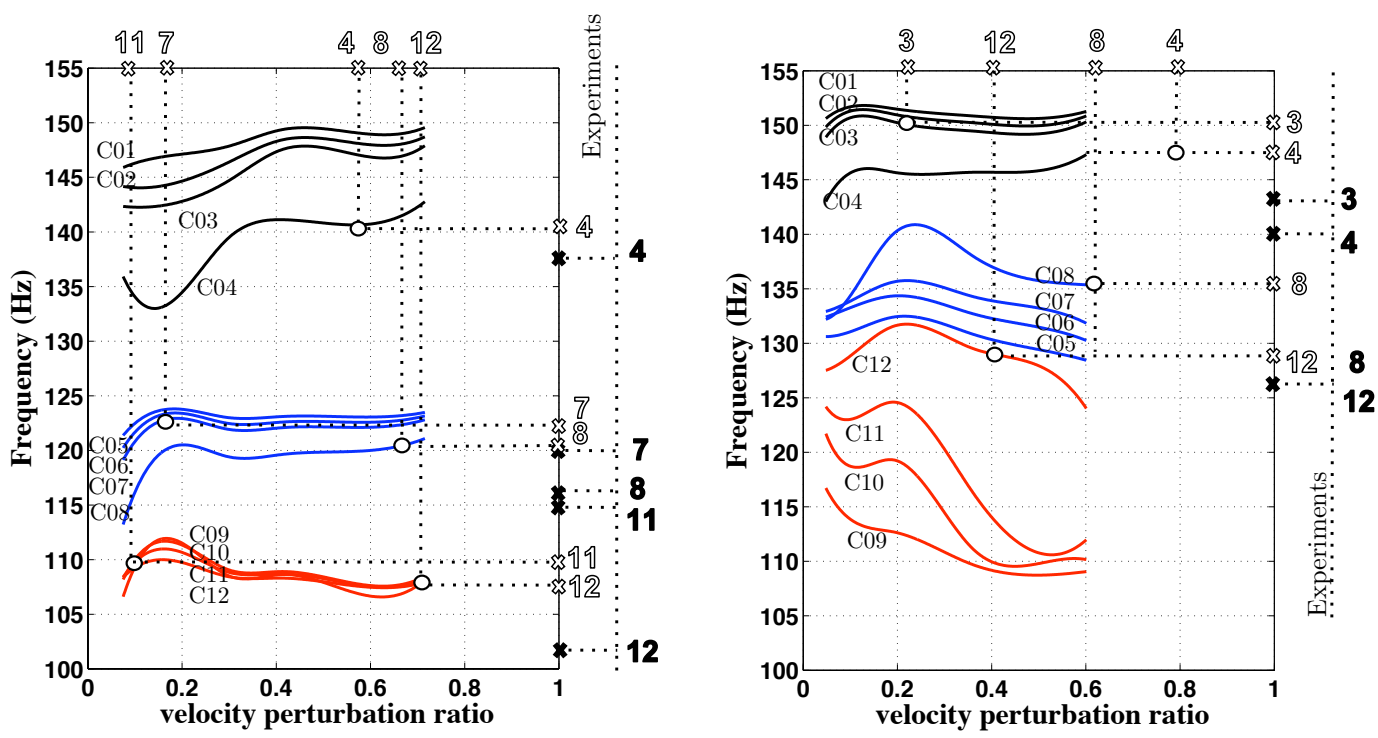

Figure 9: Evolution of the instability frequency with the velocity fluctuation level $|\hat{u}| / \bar{u}_{b}$ at the flame base. Flame A (left) and Flame B (right). White crosses indicate predictions and black crosses correspond to measured limit cycle frequencies. The corresponding predicted limit cycle is also indicated.

$\mathrm{C} 01-\mathrm{C} 04$ $\mathrm{C} 05-\mathrm{C} 08$ $\mathrm{C} 09-\mathrm{C} 12$ 
Table 6: Numerical and experimental values of the amplitude and frequency of oscillation at limit cycles.

\begin{tabular}{|c|c|c|c|c|c|c|c|c|}
\hline \multirow{3}{*}{ Case } & \multicolumn{4}{|c|}{ Flame A } & \multicolumn{4}{|c|}{ Flame B } \\
\hline & \multicolumn{2}{|c|}{ Frequency $(\mathrm{Hz})$} & \multicolumn{2}{|c|}{ Amplitude $\left(u^{\prime} / u_{b}\right)$} & \multicolumn{2}{|c|}{ Frequency $(\mathrm{Hz})$} & \multicolumn{2}{|c|}{ Amplitude $\left(u^{\prime} / u_{b}\right)$} \\
\hline & Exp. & Num. & Exp. & Num. & Exp. & Num. & Exp. & Num. \\
\hline $\mathrm{C} 01$ & - & - & - & - & - & - & - & - \\
\hline $\mathrm{C} 02$ & - & - & - & - & - & - & - & - \\
\hline $\mathrm{C} 03$ & - & - & - & - & 143 & 150 & 0.3 & 0.2 \\
\hline $\mathrm{C} 04$ & 137 & 140 & 0.8 & 0.6 & 140 & 147 & 0.9 & $\approx 0.8$ \\
\hline $\mathrm{C} 05$ & - & - & - & - & - & - & - & - \\
\hline $\mathrm{C} 06$ & - & - & - & - & - & - & - & - \\
\hline $\mathrm{C} 07$ & 120 & 122 & 0.3 & 0.2 & - & - & - & 0.1 \\
\hline $\mathrm{C} 08$ & 116 & 120 & 0.9 & 0.7 & 126 & 136 & 0.7 & 0.6 \\
\hline C09 & - & - & - & - & - & - & - & - \\
\hline $\mathrm{C} 10$ & - & - & - & - & - & - & - & - \\
\hline $\mathrm{C} 11$ & 115 & 110 & 0.4 & 0.1 & 127 & - & 0.3 & - \\
\hline $\mathrm{C} 12$ & 102 & 107 & 0.9 & 0.7 & 126 & 129 & 0.5 & 0.4 \\
\hline
\end{tabular}

carried out indicate that the values of $\omega_{i}$ estimated analytically (T1 in Tab. 4) for the twenty-four cases are slightly lower than those calculated numerically (T3 in Tab. 4) and shown previously in section 6.3. As a consequence, the respective value of the analytically estimated acoustic damping rate $\alpha$ is also lower: $\alpha_{A}^{\mathrm{av}}=79 \mathrm{~s}^{-1}$ and $\alpha_{B}^{\mathrm{av}}=98 \mathrm{~s}^{-1}$. Figure 10 shows that similar predictions are obtained when estimating oscillation amplitudes of limit cycles for Flame A by both analytical and numerical models. High levels of instability are found for configurations C04, C08 and C12 whereas weak instability is predicted in cases $\mathrm{C} 11$ and $\mathrm{C} 07$. In contrast, different observations arise when considering results for Flame B (Fig. 11), with some notably different estimations of oscillation levels. Whereas a good qualitative description of these instabilities is obtained by the Helmholtz solver, as already discussed in the previous section, the analytical model does not perform as well. The amplitude of the combustion instability in C08 and C12 is largely underestimated, as already observed in the work of Palies et al. (2011a). This is not only due to a shift to lower oscillation levels of the related trajectories, but also due to changes in shape as observed, for instance, in the C12 case. It should be noted that both analytic and numerical results are subjected to 
the same constraints (limitations) of the FDF approach and to the same difficulties that arise in estimating the damping of the system. The most likely reason for the difference between the analytical model (1D model) with respect to the numerical simulations (Helmholtz solver) is that the latter has the capability of capturing acoustic waves that are not strictly planar, and that may arise in the region close to the dump plane of the combustor. This acoustic field is closer to reality than the one obtained analytically and since it constitutes the input to the non-steady heat release one expects to obtain better results.

It will be shown in next section that differences in predictions between analytical and numerical calculations are mostly due to the geometrical simplification of the system (1D instead of 3D) and not to the distribution of the flame (represented by the gain $G$ and phase $\phi$ of the corresponding FDF) within the computational domain.

\subsection{Influence of geometrical simplifications on the growth rate trajectories}

It was shown in the previous section that for some particular trajectories (notably C09-C12 for Flame B) there is a significant difference in the prediction of the growth rate when using the analytical description with the idealized geometry ( $\mathrm{T} 1$ in Tab. 4) instead of the numerical approach based on the real geometry (T3 in Tab. 4). This section aims to demonstrate that this difference is mostly due to the $1 \mathrm{D}$ geometrical simplification in the analytical model. The configuration C12 (Tab. 1) for Flame B is chosen here because of the remarkable difference between analytical and numerical approaches (see Fig. 11). Analytical results predict a low oscillation level: C12 trajectory reaches the damping region for small velocity perturbation ratios. This is however observed neither in experiments nor in the 3D numerical results. In order to better interpret this observation, the influence of the flame distribution in the T3 computations conducted on the real geometry is analyzed. Instead of considering a ' $\mathrm{V}$ ' shaped flame, as already mentioned in section 5 and shown in Fig. 5, a planar flame is modeled as shown in Fig. 12(a). The corresponding growth rate trajectories presented in Fig. 12(b) show a small difference between simulations conducted for the V-flame and the planar flame. Contrary to what might be expected, the C12 trajectory for the planar flame keeps the same shape as the one associated to the ' $\mathrm{V}$ ' flame and is slightly shifted to higher values of growth rates. These results demonstrate that the compact flame assumption is well suited in this study and that the flame shape has a very limited influence on the results. It should be 

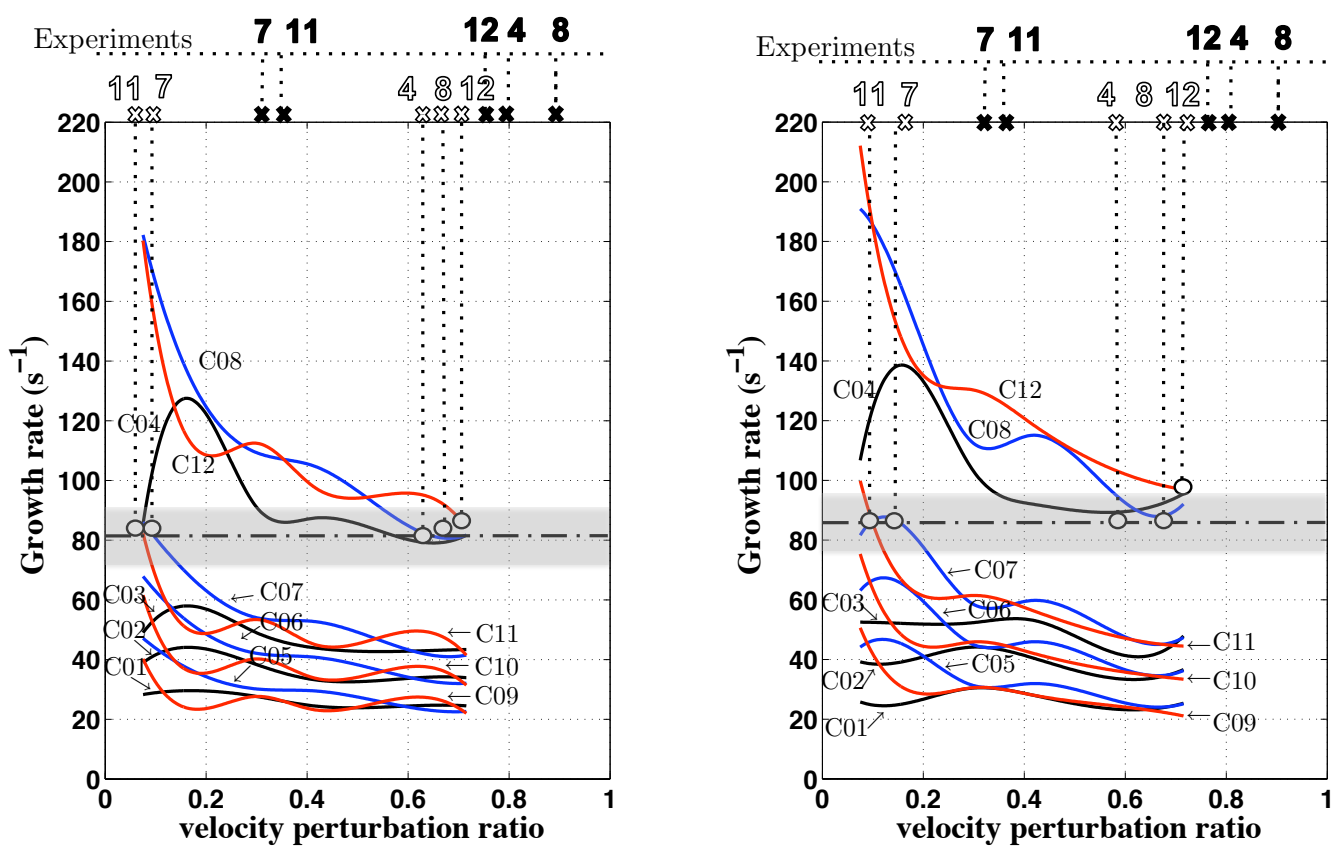

Figure 10: Growth rate trajectories evolution as a function of the velocity fluctuation level $|\hat{u}| / \bar{u}_{b}$ (Flame A). Left: analytical model predictions with the idealized 1D geometry (T1 in Tab. 4). Right: numerical simulations with the real 3D geometry (T3 in Tab. 4). White crosses indicate predictions and black crosses correspond to measured limit cycle levels. The dashed-dotted lines surrounded by the gray band indicate the region where the growth rate is balanced by damping.

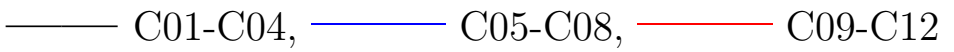



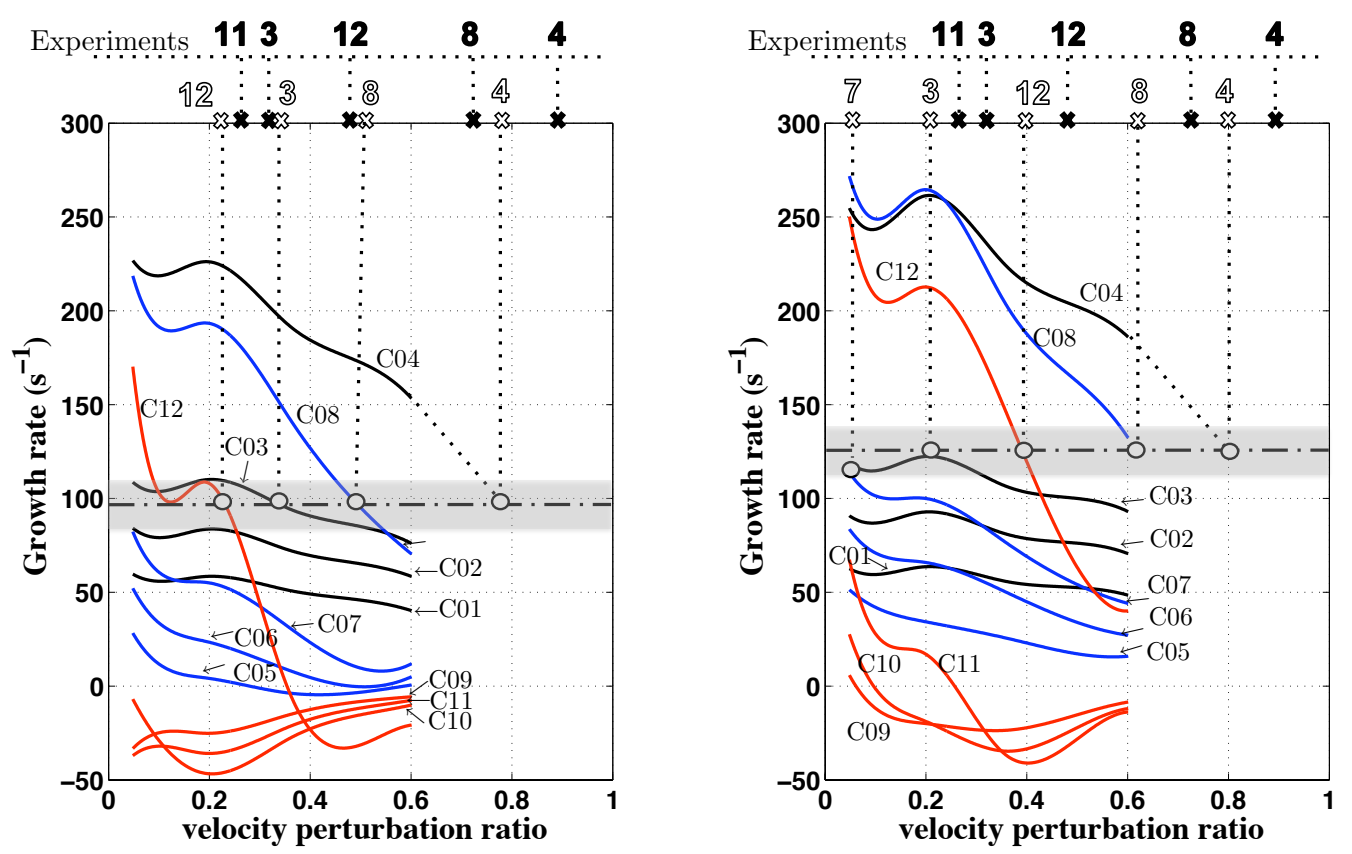

Figure 11: Growth rate trajectories evolution as a function of the velocity fluctuation level $|\hat{u}| / \bar{u}_{b}$ (Flame B). Left: analytical model predictions with the idealized 1D geometry (T1 in Tab. 4). Right: numerical simulations with the real 3D geometry (T3 in Tab. 4). White crosses indicate predictions and black crosses correspond to measured limit cycle levels. The dashed-dotted lines surrounded by the gray band indicate the region where the growth rate is balanced by damping.

$\longrightarrow$ C01-C04, - C05-C08, — C09-C12 
pointed out, nevertheless, that for non-compact flames the geometry of the combustion region should play an important role when studying combustion instabilities. In such cases, local values of the FDF obtained more readily from simulations than from experiments, become central to the prediction of instability and of limit cycle oscillations.

After considering the flame as planar, the only remaining parameter differing from analytical calculations and numerical computations is their geometry. Numerical simulations were thus conducted with the same idealized 1D geometry and with a compact planar flame as in the analytical approach. These simulations are designated by T2 in Tab. 4. As expected no significant difference between $\mathrm{T} 1$ and $\mathrm{T} 2$ calculations is observed as illustrated in Fig. 12(b). As a result, it could be stated that resolving the acoustics on the actual geometry of the combustion system may become crucial for a good estimation of growth rate trajectories of combustion instabilities.

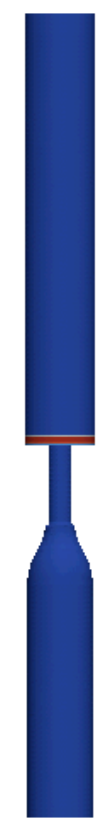

(a) Planar Flame (in red) in 'T3' computations

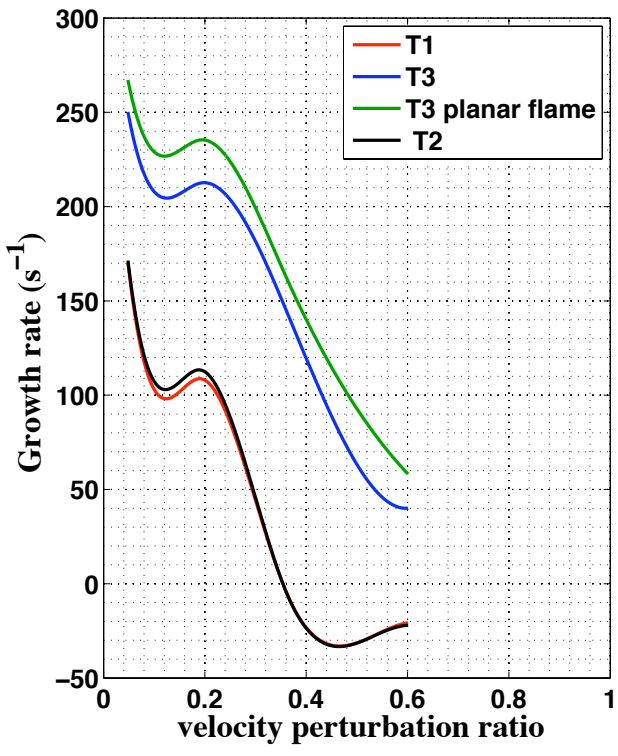

(b) Growth rate trajectories for C12 - Flame B

Figure 12: Influence of geometrical simplifications on the growth rate trajectories calculated for the C12 configuration. Flame B. See Tab. 4 for labeling. 


\section{Conclusions}

Analysis of the thermo-acoustic behavior of a swirled premixed combustor has been carried out in a systematic manner by considering a large number of geometrical configurations and two operating conditions. It has been demonstrated that a numerical tool based on the Helmholtz equation and the Arnoldi algorithm for solving the corresponding eigenvalue problem can be combined with the FDF methodology in order to estimate growth rate values of acoustic modes, limit cycle frequencies and amplitudes. Some convergence problems can arise when solving the nonlinear eigenvalue problem, but they can be overcome if the fixed point method is applied under relaxation constraints. This numerical procedure has been validated by comparing the corresponding results to an analytical description using a simplified geometry. The Helmholtz solver is then used to compute the complex eigenfrequencies of the exact geometry of the combustor. The stability of the burner is then evaluated and predictions are found to agree in almost all cases with the experimental behavior of the combustion system. Finally, the growth rate trajectories of the fundamental mode of the system are estimated by the computational tool. The amplitude of the limit cycles obtained for the linearly unstable operating points are then assessed by equating the computed growth rates to the measured acoustic damping of the combustor.

The effect of the flame shape is encompassed in the FDF under the assumption that the flame is compact (i.e., that the acoustic wavelength is large compared to the flame size). This has been checked in many previous analytical studies which commonly use a thin discontinuity to represent the flame. The comparison carried out in this article supports this idealization. The flame shape becomes important at higher frequencies when the wavelength is commensurate with the flame size. It is then important to consider that unsteady combustion is distributed and one has to resort to a local description of the flame response.

One uncertainty is that of assessing the damping of the system but this can be tackled by combining measurements of the resonant response of the system with calculations of the flame contribution. Using this idea, a fair agreement was found between the observed and the computed limit cycle amplitudes.

\section{Acknowledgments}

Support provided by the ANR MICCA project is gratefully acknowledged. 


\section{References}

Balachandran, R., Ayoola, B. O., Kaminski, C. F., Dowling, A. P., Mastorakos, E., 2005. Experimental investigation of the nonlinear response of turbulent premixed flames to imposed inlet velocity oscillations. Combust. Flame 143, 37-55.

Bellows, B. D., Bobba, M. K., Forte, A., Seitzman, J. M., Lieuwen, T., 2007. Flame transfer function saturation mechanisms in a swirl-stabilized combustor. Proc. Combust. Inst.31, 3181-3188.

Bloxsidge, G. J., Dowling, A. P., Langhorne, P. J., 1988. Reheat buzz: an acoustically coupled combustion instability. part 2. theory. J. Fluid Mech.193, 445-473.

Boudy, F., Durox, D., Schuller, T., Candel, S., 2011. Nonlinear mode triggering in a multiple flame combustor. Proc. Combust. Inst.33, 1121-1128.

Camporeale, S. M., Fortunato, B., Campa, G., 2011. A finite element method for three-dimensional analysis of thermo-acoustic combustion instability. J. Eng. Gas Turb. and Power133, 011506.

Candel, S., 2002. Combustion dynamics and control: Progress and challenges. Proc. Combust. Inst.29, 1-28.

Crocco, L., 1951. Aspects of combustion instability in liquid propellant rocket motors. Part I. J. American Rocket Society21, 163-178.

Crocco, L., 1952. Aspects of combustion instability in liquid propellant rocket motors. Part II. J. American Rocket Society22, 77-16.

Crocco, L., 1969. Research on combustion instability in liquid propellant rockets. Proc. Combust. Inst.12, 85-99.

Culick, F. E. C., 1994. Some recent results for nonlinear acoustic in combustion chambers. AIAA Journal32 (1), 146-169.

Dowling, A. P., 1997. Nonlinear self-exited oscillations of a ducted flame. J. Fluid Mech.346, 271-290.

Dowling, A. P., 1999. A kinematic model of ducted flame. J. Fluid Mech.394, $51-72$. 
Dowling, A. P., Stow, S. R., 2003. Acoustic analysis of gas turbine combustors. J. Prop. Power19 (5), 751-764.

Duchaine, F., Boudy, F., Durox, D., Poinsot, T., 2011. Sensitivity analysis of transfer functions of laminar flames. Combust. Flame 158 (12), 2384-2394.

Ducruix, S., Durox, D., Candel, S., 2000. Theoretical and experimental determinations of the transfer function of a laminar premixed flame. Proc. Combust. Inst.28, 765-773.

Durox, D., Schuller, T., Noiray, N., Candel, S., 2009. Experimental analysis of nonlinear flame transfer functions for different flame geometries. Proc. Combust. Inst.32, 1391-1398.

Huber, A., Polifke, W., 2009. Dynamics of practical premix flames. part I: Model structure and identification. Int. J. of Spray and Combustion Dynamics 1 (2), 199-229.

Kim, K., Hochgreb, S., 2011. The nonlinear heat release response of stratified lean-premixed flames to acoustic velocity oscillations. Combust. Flame $158,2482-2499$.

Kim, K., Lee, J., Quay, B., Santavicca, D., 2010. Spatially distributed flame transfer functions for predicting combustion dynamics in lean premixed gas turbine combustors. Combust. Flame 157, 1718-1730.

Külsheimer, C., Büchner, H., 2002. Combustion dynamics of turbulent swirling flames. Combust. Flame 131, 70-84.

Lieuwen, T., Yang V., (Eds.), 2005. Combustion instabilities in gas turbine engines: operational experience, fundamental mechanisms and modeling. Prog. in Astronautics and Aeronautics AIAA 210.

Moeck, J. P., Bourgouin, J. F., Durox, D., Schuller, T., Candel, S., 2012. Nonlinear interaction between a precessing vortex core and acoustic oscillations in a turbulent swirling flame. Combust. Flame 159, 2650-2668.

Nicoud, F., Benoit, L., Sensiau, C., 2007. Acoustic modes in combustors with complex impedances and multidimensional active flames. AIAA Journal45, 426-441. 
Noiray, N., Durox, D., Schuller, T., Candel, S., 2008. A unified framework for nonlinear combustion instability analysis based on the flame describing function. J. Fluid Mech.615, 139-167.

Palies, P., Durox, D., Schuller, T., Candel, S., 2010. The combined dynamics of swirler and turbulent premixed swirling flames. Combust. Flame 157, 1698-1717.

Palies, P., Durox, D., Schuller, T., Candel, S., 2011a. Nonlinear combustion instabilities analysis based on the flame describing function applied to turbulent premixed swirling flames. Combust. Flame 158, 1980-1991.

Palies, P., Durox, D., Schuller, T., Morenton, P., Candel, S., 2009. Dynamics of premixed confined swirling flames. C. R. Mecanique 337, 395-405.

Palies, P., Schuller, T., Durox, D., Candel, S., 2011b. Modeling of premixed swirling flames transfer functions. Proc. Combust. Inst.33, 2967-2974.

Palies, P., Schuller, T., Durox, D., Gicquel, L., Candel, S., 2011c. Acoustically perturbed turbulent premixed swirling flames. Phys. Fluids23 (1), 037101.

Poinsot, T., Veynante, D., 2005. Theoretical and numerical combustion. R. T. Edwards.

Rayleigh, L., 1878. The explanation of certain acoustic phenomena. Nature July 18, 319-321.

Roux, S., Lartigue, G., Poinsot, T., Meier, U., Bérat, C., 2005. Studies of mean and unsteady flow in a swirled combustor using experiments, acoustic analysis and large-eddy simulations. Combust. Flame 141, 40-54.

Sattelmayer, T., Polifke, W., 2003. Assessment of methods for the computation of the linear stability of combustors. Combust. Sci. Tech.175 (3), $453-476$.

Schimek, S., Moeck, J. P., Paschereit, C. O., 2011. An experimental investigation of the nonlinear response of an atmospheric swirl-stabilized premixed flame. J. Eng. Gas Turb. and Power133 (10), 101502. 
Schuermans, B., Guethe, F., Pennell, D., Guyot, D., Paschereit, C. O., 2011. Thermoacoustic modeling of a gas turbine using transfer functions measured under full engine pressure. J. Eng. Gas Turb. and Power132 (11), 11503 .

Schuller, T., Durox, D., Palies, P., Candel, S., 2012. Acoustic decoupling of longitudinal modes in generic combustion systems. Combust. Flame 159 (5), 1921-1931.

Tay-Wo-Chong, L., Bomberg, S., Ulhaq, A., Komarek, T., Polifke, W., 2012. Comparative validation study on identification of premixed flame transfer function. J. Eng. Gas Turb. and Power134 (2), 021502-1-8.

Trefethen, L. N., Bau, D., 1997. Numerical linear algebra. Society for Industrial and Applied Mathematics SIAM., Philadelphia, United States of America.

Truffin, K., Poinsot, T., 2005. Comparison and extension of methods for acoustic identification of burners. Combust. Flame 142 (4), 388-400. 